\title{
Cardiac sarcoidosis: two case reports.
}

\author{
Felix Awindaogo ${ }^{1}$, Jane Afriyie-Mensah ${ }^{2}$, Harold Ayetey $^{3}$, and Emmanuella Tagoe ${ }^{1}$ \\ ${ }^{1}$ Korle Bu Teaching Hospital \\ ${ }^{2}$ University of Ghana Medical School \\ ${ }^{3}$ University of Cape Coast
}

December 18, 2020

\begin{abstract}
The clinical presentation of cardiac sarcoidosis is variable. We report two cases of cardiac sarcoidosis to highlight the varied clinical presentations and diagnostic challenges in our setting and encourage the consideration of sarcoidosis as a differential in unexplained arrhythmias and heart failure.
\end{abstract}

\section{CASE REPORT}

ARTICLE TITLE

Cardiac sarcoidosis: two case reports

\section{Author names}

${ }^{1}$ Felix Razak Awindaogo MD, MSc

${ }^{2}$ Jane Sandra Afriyie-Mensah MD

${ }^{3}$ Harold Ayetey MD, PhD

${ }^{1}$ Emmanuella Naa Deeidei Tagoe MD

\section{Author Affiliation}

${ }^{1}$ Department of Medicine and Therapeutics, Korle Bu Teaching Hospital, Accra, Ghana ${ }^{2}$ Department of Medicine and Therapeutics, University of Ghana Medical School, Accra, Ghana ${ }^{3}$ Department of Internal Medicine and Therapeutics, University of Cape Coast, Cape Coast, Ghana

\section{Correspondence}

Felix Razak Awindaogo

Department of Medicine and Therapeutics

Korle Bu Teaching Hospital

Accra, Ghana

Email: fawindaogo@yahoo.com

\section{ABSTRACT}

The clinical presentation of cardiac sarcoidosis is variable. We report two cases of cardiac sarcoidosis to highlight the varied clinical presentations and diagnostic challenges in our setting and encourage the consideration of sarcoidosis as a differential in unexplained arrhythmias and heart failure. 


\section{KEYWORDS}

sarcoidosis, bundle branch block, bradycardia, heart block, heart failure

\section{KEY CLINICAL MESSAGE}

Cardiac sarcoidosis is a major cause of death and disability in sarcoid patients. It can be benign and asymptomatic or life-threatening.

Early screening is crucial, since prompt therapy may lead to resolution of cardiac arrhythmias or dysfunction.

\section{INRODUCTION}

Sarcoidosis is a multisystem inflammatory disease characterized by the presence of noncaseating granulomas of unknown aetiology. Although the disease affects almost every organ system in the body, lung involvement occurs in over $90 \%$ of all cases. ${ }^{1}$ The estimated prevalence of sarcoidosis is $20-60$ cases per 100,000 with worse outcomes among African-American populations. ${ }^{1}$ There is lack of data on the incidence and disease burden of sarcoidosis in Africa and Ghana. Detailed clinical and epidemiological studies are urgently required in this area.

Sarcoidosis has the worst prognosis when it involves the heart muscle. Cardiac sarcoid (CS) is estimated to account for more than two-thirds of global deaths in sarcoidosis patients. ${ }^{2,3}$ Heart muscle involvement may be isolated (with no overt clinical manifestations of systemic sarcoidosis masking early disease detection) or in association with other systems involvement particularly pulmonary sarcoidosis. The estimated prevalence of cardiac sarcoidosis (CS) is between 20 to $30 \%$ in patients with sarcoidosis from autopsy studies as well as screening of known sarcoidosis patients with cardiac magnetic resonance imaging (MRI). However, only $5 \%$ of patients with systemic sarcoidosis have clinical evidence of cardiac sarcoidosis. ${ }^{3-5}$ The prevalence of isolated cases of CS is unclear although there are case reports of such occurrence in the literature. ${ }^{6}$

Clinical presentation of CS is variable and can be benign and asymptomatic or life-threatening with symptoms of heart failure, cardiac arrhythmias or sudden cardiac death. Mitral valve regurgitation due to papillary muscle dysfunction, pericardial involvement manifesting as pericarditis or tamponade and pulmonary hypertension due to pulmonary parenchyma injury or left ventricle (LV) dysfunction are all reported manifestations of cardiac sarcoidosis. ${ }^{3,7}$ CS has also been identified incidentally after ECG abnormalities and cardiac symptoms in patients with sarcoidosis, or unfortunately following sudden death. ${ }^{3,6}$ Indeed up to $50 \%$ of deaths in sarcoidosis may be related to cardiac involvement. ${ }^{8}$

The diagnosis of cardiac sarcoidosis however remains a challenge because of non-specific symptoms and the absence of a single examination technique to reliably diagnose the condition. Screening is however imperative since treatment can significantly reduce the risk of sudden death among affected patients. ${ }^{9}$ Expert opinion recommend clinicians actively enquire about cardiac symptoms in sarcoidosis patients at follow-up visits and have repeat ECGs every 6 to 12 months and particularly during tapering of corticosteroid treatment. Detailed evaluation is warranted if clinical or ECG abnormalities are detected. . $^{3,10}$

Several established diagnostic guidelines in existence are based on expert consensus with none validated by prospective data or clinical trials. ${ }^{11}$ Clinicians must combine clinical symptoms with advanced cardiac imaging techniques consistent with sarcoidosis and demonstrate histological presence of noncaseating granulomas, rule out alternative diagnosis and causes of noncaseating granulomas before making a diagnosis of cardiac sarcoidosis. $^{4,12}$

The patchy distribution of noncaseating granulomas in myocardium contributes to the low diagnostic yield (sensitivity of 10-25\%) of endomyocardial biopsy despite being the gold standard of diagnosis. ${ }^{13}$ The invasiveness of the procedure and its attendant complications contribute to the limited usefulness of endomyocardial biopsy. ${ }^{14}$ CS may be accompanied by LV dilatation as well as regional and global wall motion abnormalities. Regional granulomatous inflammation can also lead to wall thinning and akinesis. Cardiac magnetic resonance (CMR), currently the investigation of choice can reliably identify all of these abnormalities by 
utilising a combination of morphological and functional assessment tools that lead to excellent sensitivity $(96.9 \%)$ and specificity $(100 \%)$ for detecting cardiac sarcoidosis. ${ }^{3,15-17}$

Fluorodeoxyglucose - positron emission tomography (FDG-PET) scan is a useful nuclear imaging modality that employs radioactive glucose to detect areas of active inflammation with a sensitivity of $89 \%$ and specificity of $78 \%$ in detecting cardiac sarcoidosis. FDG-PET scan has the benefit of demonstrating both perfusion and metabolic activity aiding in diagnostic specificity. ${ }^{18}$ However, FDG-PET is even less accessible in settings such us ours.

The ECG is very useful in identifying conduction disorders, arrhythmias or paroxysmal and intermittent rhythm disturbances in the case of Holter ECG. However its sensitivity and specificity of diagnosing CS is low. Echocardiography, although useful, cannot establish the diagnosis of CS but its importance is seen in the assessment of associated complications and monitoring of the course of the disease. ${ }^{16,18}$

The management of CS is multidisciplinary and involves cardiologists, pulmonologists, radiologists, rheumatologists and others. The pharmacological management of cardiac sarcoidosis involves the use of immunosuppressive medications and cardiac-specific therapy to manage cardiac sequelae of sarcoidosis. The goal of immunosuppressive therapy is to reverse any ongoing active granulomatous myocarditis and potentially prevent the progression of myocardial granulomatous inflammation to scar tissue which leads to arrhythmias and ventricular dysfunction. ${ }^{18}$ As the most frequent cause of death from CS are heart rhythm disorders such as AV block and ventricular arrhythmias, an irreplaceable role in the management of CS patients is pacemakers and implantable cardiac defibrillators (ICDs). ${ }^{19}$

Beyond these, general cardiology inputs in the management of the CS patient may include guideline directed medical management of systolic and diastolic heart failure as with other aetiologies of heart failure. The caveat however is use of beta blockers in the absence of pacemaker because of frequent AV conduction abnormalities. ${ }^{20}$ Antiarrhythmic agents including amiodarone may be used to maintain normal sinus rhythm in patients with atrial arrhythmias or symptomatic ventricular tachycardia. ${ }^{20}$

Cardiac specific treatment typically occurs in combination with immunosuppressants since they have no impact on the ongoing pathological process of sarcoidosis. ${ }^{3}$ Systemic corticosteroids are the cornerstone of the treatment in CS and are considered first-line agents because their efficacy and attainment of significant response in a relatively short period. Other immunosuppressive drugs such as methotrexate, azathioprine, mycophenolate mofetil, leflunomide, cyclosporine, or cyclophosphamide can be used in the event of corticosteroids failure, development of adverse side effects with corticosteroid therapy, prevention of corticosteroid adverse side effects in patients requiring higher doses ( $>10 \mathrm{mg} /$ day prednisolone) of corticosteroids as maintenance therapy and in combination therapy. ${ }^{3,20,21}$

We present two cases of cardiac sarcoidosis with differing clinical presentations.

\section{CASE REPORTS}

\section{Case 1}

A previously well 58 year old woman with an unremarkable medical history was seen at the respiratory clinic with a six month history of a dry cough, easy fatigability and progressive breathlessness on exertion. She was unaware of any precipitants and had received numerous antibiotic courses, salbutamol and budesonide inhalers, and prednisolone with mild improvement of her symptoms. Physical examination including chest auscultation was normal except for a regular tachycardia of 106 beats/min. Chest $\mathrm{x}$ - ray (CXR) revealed a bilateral hilar lymphadenopathy and cardiomegaly. A contrast - enhanced computed tomography (CT) scan of the chest showed bilateral reticulonodular opacities with bilateral hilar lymphadenopathy suggestive of stage II pulmonary sarcoidosis. An electrocardiogram (ECG) showed sinus tachycardia with a left bundle branch block (Figure 1) and a transthoracic echocardiogram revealed a dilated left ventricle with severely reduced LV systolic function (ejection fraction of 30\%) and dyssynchrony of the interventricular septum (IVS). Laboratory investigations showed elevated serum angiotensin converting enzyme (ACE) of $69.6 \mathrm{UI} / \mathrm{L}$ $(8.0-52.0)$, erythrocyte sedimentation rate (ESR) of $33 \mathrm{mmfall} / \mathrm{hr}(3.0-5.0)$ and hypercalcaemia of 2.79 
mmol/L (2.12 - 2.62). ANA was negative and sputum for GeneXpert (GeneXpert Dx System Version 4.8) did not detect Mycobacterium tuberculosis DNA. Lung function tests revealed a restrictive pattern with a forced expiratory volume in the first second (FEV1) of 1.44L (predicted, 2.18L), a forced vital capacity (FVC) of $1.88 \mathrm{~L}$ (predicted, 2.51L) and a FEV1/FVC ratio of $77 \%$ (predicted, $87 \%$ ). A diagnosis of pulmonary sarcoidosis was made with possible cardiac involvement and steroid therapy initiated with good improvement of symptoms within 3 months. The patient developed symptoms of heart failure (orthopnoea, paroxysmal nocturnal dyspnoea, bilateral leg swelling) 6 months later when her steroid dose was being tapered. She was admitted and managed for heart failure (NYHA Class III). Cardiac MRI done later showed a moderately dilated LV with moderately reduced ejection fraction (EF) of $40 \%$, discoordinate movement of interventricular septum and a thinned and hypokinetic apical anterior wall. There was no active myocardial inflammation or oedema but there was midwall late gadolinium enhancement of the basal inferior LV wall, the basal lateral LV wall with patchy near-transmural enhancement of the apical anterior and mid to apical lateral LV wall (Figure 2) all suggestive of cardiac sarcoid. The prednisolone dose was increased to $1 \mathrm{mg} / \mathrm{kg}$ and azathioprine added to allow for prednisolone dose tapering. She remains stable on above in addition to heart failure therapy (NYHA class I). Her ECG findings remain unchanged.

\section{Case 2}

A 51 year old male, with no chronic illnesses was seen with a 4 month history of intermittent dry cough, palpitations and progressive shortness of breath on exertion. He had received treatment for recurrent chest infections and was a lifelong non-smoker. He was referred to the respiratory clinic with an abnormal CXR. Clinical examination revealed bilateral lower lung zones crepitations, $\mathrm{SpO}_{2}$ of $94 \%$ at rest and on room air with exertional drop to $88 \%$. He had a regular bradycardia of 35 beats/min and blood pressure of 137/74 mmHg. ECG revealed a third degree heart block (Figure 3). His transthoracic echocardiogram showed normal LV morphology (relative wall thickness, 0.32; LV mass index, $91 \mathrm{~g} / \mathrm{m}^{2}$ ); normal systolic and diastolic function, and normal segmental wall motion. There was complete atrioventricular block with junctional escape rhythm as well as intermittent Mobitz type I or 2 block on his 24 hour Holter ECG. A high resolution chest CT-Scan showed ground glass opacification with multiple tiny nodules in the mid and lower lung zones, increased reticulation and mediastinal lymphadenopathy suggestive of Stage II sarcoidosis. Serum ACE was elevated; $116.0 \mathrm{IU} / \mathrm{L}(8.0-52.0)$ and pulmonary function test showed a restrictive pattern with a FEV1 of 2.23L (predicted, 3.64), FVC of 2.53L (predicted, 4.68) and a FEV1/FVC ratio of $88.3 \%$ (predicted, $77.7 \%$ ). A diagnosis of pulmonary sarcoidosis with probable cardiac involvement was made and confirmed by Cardiac MRI which revealed Late Gadolinium enhancement of the mid-septum, basal to mid-septum and lateral LV wall with corresponding oedema and inflammation in STIR images highly suggestive of cardiac sarcoidosis (Figure 4).

Patient was started on corticosteroid and azathioprine and assessed for a possible pacemaker insertion which patient refused. He however showed marked improvement in the respiratory symptoms and a conversion of the complete heart block to a first degree heart block after 8 weeks of therapy (Figure 5). He is being followed up periodically with ECGs.

\section{DISCUSSION}

Cardiac sarcoidosis is the most ominous manifestation of sarcoidosis accounting for as much as $50 \%$ of deaths in patients with sarcoidosis. ${ }^{8}$ The prevalence of cardiac involvement amongst patients with systemic sarcoidosis varies widely for unknown reasons, but ranges between 3.7 to $54.9 \%$ depending upon the imaging techniques used and the population studied., ${ }^{4,14}$ There is scanty data on CS in our setting and in Africa as a whole with no case reports identified. The triad of cardiac manifestations are conduction abnormality, ventricular tachycardia and heart failure.

CS though often clinically silent is frequently fatal. It's wide spectrum of clinical manifestations arise from the variable locations of sarcoid lesions in the myocardium. ${ }^{4}$ There is however a predilection for the base of the interventricular septum, the conduction system and the left ventricular (LV) free wall, although, other parts of heart including the papillary muscles, right ventricular wall, atria, valve leaflets, intramyocardial vessels, 
pericardium and endocardium may be involved. ${ }^{3,7,22}$ Indeed, both patients reported here had involvement of the base of the interventricular septum, the conduction system and the left ventricular (LV) free wall.

Conduction disease as observed in our report, is the most common electrophysiologic manifestation of CS with a prevalence up to $62 \% .{ }^{14}$ Complete heart block and bundle branch blocks have been reported in 23$30 \%$ and $12-32 \%$, respectively of patients with myocardial sarcoidosis. ${ }^{7}$ Advanced AV blocks may lead to syncope or sudden cardiac death, warranting inclusion of sarcoidosis as a differential in the evaluation of patients with syncope, especially when it occurs in young and middle-aged patients. Studies suggest CS may be accountable for unexplained second or third degree atrioventricular (AV) block in patients less than 60 years old ${ }^{14,23}$. Indeed, implantable cardioverter-defibrillators (ICDs) are recommended for CS patients with complete heart block, prior ventricular arrhythmias, cardiac arrest or LV ejection fraction of $<35 \% .{ }^{24}$

A prospective evaluation of patients aged 18 to 60 years presenting with unexplained Mobitz II or 3rd degree AVB and no previous history of sarcoidosis by Nery et al found that $34 \%$ of patients had CS and were more likely to experience major adverse cardiac events. ${ }^{25}$ Data from the National Inpatient Sample (NIS) database from 2012-2014 has shown that complete heart block is more prevalent among patients with sarcoidosis compared to those without a diagnosis of sarcoidosis and increased the odds of developing complete heart block among other medical comorbidities like coronary artery disease, age, gender and heart failure. $^{26}$

A recent Finnish study of 325 cases of cardiac sarcoidosis from the Myocardial Inflammatory Diseases in Finland Study Group Registry diagnosed from 1988 to 2015, reports that 143 (44\%) patients presented with Mobitz II second degree or third degree AVB in the absence of other explanatory cardiac disease. ${ }^{27}$ Likewise, another Finnish study of 72 patients aged $<55$ presenting with new-onset, unexplainable Mobitz II second degree or third degree AVB, 14 of 72 (19\%) patients had histologically confirmed CS whilst another $4(6 \%)$ had giant cell myocarditis. ${ }^{23}$

Bundle branch blocks are also a frequent occurrence in CS patients. Our first case had left bundle branch block (LBBB) which is known to be less common compared to right bundle branch blocks (RBBB). ${ }^{7,28,29}$ Schuller et al found that among a cohort of patients with biopsy - proven pulmonary sarcoidosis a BBB pattern is associated with cardiac involvement ${ }^{29}$ and RBBB pattern was more prevalent than LBBB in the CS population in a Danish study. ${ }^{28}$

Cardiac sarcoidosis is frequently overlooked as the underlying cause of heart failure and accounts for 25 $75 \%$ of cardiac deaths in patients with cardiac sarcoidosis. Left ventricular systolic and diastolic dysfunction leading to progressive congestive heart failure often occurs due to conduction or rhythmic abnormalities, or as a result of extensive myocardial infiltration or both. .,10,30 A study of core LV biopsies obtained during LV assist device implantation showed granulomatous myocarditis consistent with sarcoidosis in previously undiagnosed CS in 6 of 177 mixed cardiomyopathy patients (3.4\%). ${ }^{31}$ In an examination of explanted hearts, Roberts et al found that among 130 heart transplants with a clinical diagnosis of non-ischaemic cardiomyopathy, $8(6.2 \%)$ had undiagnosed CS. ${ }^{32}$

Due to the low sensitivity of endomyocardial biopsy, cardiac MRI has become the investigation of choice for cardiac sarcoidosis in the patient with a high clinical suspicion. As recommended by experts' consensus, clinical symptoms with consistent advanced cardiac MRI findings should be combined with histological presence of noncaseating granulomas demonstrated in biopsy of other sites of involvement such as the skin or lungs. In both cases presented, the presence of cardiac complications prevented biopsy of lung tissue via CT-guided biopsy as endobronchial ultrasound transbronchial needle aspiration (EBUS-TBNA) is not available in our practice. High resolution CT-scan (HRCT) of the chest which has significant correlation with histology in the diagnosis of pulmonary sarcoidosis was highly suggestive in both cases and supported by some laboratory markers such as serum ACE level and hypercalcaemia. ${ }^{33}$

Broadly, current treatment for CS involves immunosuppression and antiarrhythmic agents for inflammation and antiarrhythmic drugs and/or ablation for scar formation/fibrosis. Optimal duration of treatment remain controversial, but corticosteroids and other immunosuppressive agents improve cardiac function, prevent 
further formation of granulomas, and reduce arrhythmias and incidence of sudden cardiac death as depicted in the cases presented, but patients with advanced cardiac dysfunction (LV ejection fraction <30\%) may remain unresponsive to corticosteroid therapy. ${ }^{3,15,34-36}$ Even though corticosteroids remain the cornerstone in the treatment of clinically active or symptomatic CS, dosing, selection and timing and duration of therapy for cardiac sarcoidosis remains controversial. ${ }^{20,36}$ Late gadolinium enhancement in Cardiac MRI has emerged as an important tool for risk stratification for sudden cardiac death (SCD) in CS and is of key importance in the decision for ICD implantation in CS patients considered at high risk for SCD. Although both patients in this report had LGE on their cardiac MRI scans for which an ICD was indicated, this service was not available in our country.

\section{CONCLUSION}

We report two patients with cardiac sarcoidosis who initially presented with conduction disease and heart failure. The diagnosis of CS in both patients had been delayed because of delay in diagnosing sarcoidosis and not establishing a link between AV arrhythmia and sarcoidosis. The recent availability of cardiac MRI in Ghana allowed the diagnosis to be confirmed. Although relatively uncommon, cardiac sarcoidosis should be included in the differentials of unexplained conduction disease or arrhythmias as optimal management will prevent cardiac dysfunction and sudden cardiac deaths.

\section{ACKNOWLEDGEMENT}

No acknowledgments to be made.

\section{CONFLICT OF INTEREST}

No conflict of interest.

\section{AUTHOR CONTRIBUTION}

Felix Razak Awindaogo: Conceptualization; writing-original draft; writing-review and editing. Jane Sandra Afriyie-Mensah:Conceptualization, writing-review and editing. Harold Ayetey:Writing- review and editing; analysis and interpretation of cardiac MRI images. Emmanuella Naa Deedei Tagoe: Writing-original draft (supporting); writing-review and editing.

All authors were involved in editing and approval of manuscript.

\section{ETHICAL APPROVAL}

Consent obtained from patients.

\section{DATA AVAILABILITY STATEMENT}

We will make available data (images and reports) that support our report upon request.

\section{ORCHID}

Felix Razak Awindaogo https://orcid.org/0000-0002-2612-2685

\section{REFERENCES}

1. J. Larry Jameson, Dennis L. Kasper, Dan L. Longo, Anthony S. Fauci, Stephen L. Hauser JL, ed. Disorders of Immune-Mediated Injury. In:Harrison's Principles of Internal Medicine . 20th Editi. New York : McGraw-Hill Education; 2018:2600-2607.

2. Yahalom M, Koren O, Turgeman Y. High Degree Atrioventricular Block Complicated Cardiac Sarcoidosis : Case Report. 2018:157-163. doi:10.4236/wjcs.2018.89015

3. Nunes H, Freynet O, Naggara N, et al. Cardiac Sarcoidosis.Semin Respir Crit Care Med . 2010;31(04):428441. doi:10.1055/s-0030-1262211 
4. Hulten E, Aslam S, Osborne M, Abbasi S, Bittencourt MS, Blankstein R. Cardiac sarcoidosis-state of the art review. Cardiovasc Diagn Ther . 2016;6(1):50-63. doi:10.3978/j.issn.2223-3652.2015.12.13

5. Jotterand M, Grabherr S, Lobrinus JA, Michaud K. Sudden cardiac death and sarcoidosis of the heart in a young patient. Cardiovasc Pathol . 2017;28:18-20. doi:10.1016/j.carpath.2017.01.003

6. Kandolin R, Lehtonen J, Graner M, et al. Diagnosing isolated cardiac sarcoidosis. J Intern Med . 2011;270(5):461-468. doi:10.1111/j.1365-2796.2011.02396.x

7. Sekhri V, Sanal S, Delorenzo LJ, Aronow WS, George P. Review paper Cardiac sarcoidosis : a comprehensive review. 2011. doi:10.5114/aoms.2011.24118

8. Perry A, Vuitch F. Causes of death in patients with sarcoidosis: A morphologic study of 38 autopsies with clinicopathologic correlations. Arch Pathol Lab Med . 1995;119(2):167-172.

9. Judson MA. The diagnosis of sarcoidosis. Curr Opin Pulm Med . 2019;25(5):484-496. doi:10.1097/MCP.0000000000000596

10. Birnie DH, Nery PB, Ha AC, Beanlands RSB. Cardiac Sarcoidosis. 2016;68(4). doi:10.1016/j.jacc.2016.03.605

11. Judson MA. Screening sarcoidosis patients for cardiac sarcoidosis: What the data really show. Respir Med . 2019;154:155-157. doi:10.1016/j.rmed.2019.05.007

12. Tan JL, Fong HK, Birati EY, Han Y. Cardiac Sarcoidosis. Am J Cardiol . 2019;123(3):513-522. doi:10.1016/j.amjcard.2018.10.021

13. Evanchan JP, Crouser ED. Cardiac Sarcoidosis : Recent Advances in Diagnosis and Treatment and an Argument for the Need for a Systematic Multidisciplinary Approach to Management. 2013;4(March):11601174. doi:10.19102/icrm.2013.040308

14. Selan JC, Michaelson M, Fanburg BL, Estes NAM. Evaluation and management of heart rhythm disturbances due to cardiac sarcoidosis. Hear Lung Circ . 2014;23(12):1100-1109. doi:10.1016/j.hlc.2014.07.065

15. Orii M, Tanimoto T, Ota S, et al. Diagnostic accuracy of cardiac magnetic resonance imaging for cardiac sarcoidosis in complete heart block patients implanted with magnetic resonance-conditional pacemaker. $J$ Cardiol . 2020;(2019). doi:10.1016/j.jjcc.2020.02.014

16. Kouranos V, Tzelepis GE, Rapti A, et al. Complementary Role of CMR to Conventional Screening in the Diagnosis and Prognosis of Cardiac Sarcoidosis. JACC Cardiovasc Imaging . 2017;10(12):1437-1447. doi:10.1016/j.jcmg.2016.11.019

17. Stanton KM, Ganigara M, Corte P, et al. The Utility of Cardiac Magnetic Resonance Imaging in the Diagnosis of Cardiac Sarcoidosis.Hear Lung Circ . 2017;26(11):1191-1199. doi:10.1016/j.hlc.2017.02.021

18. Hamzeh N, Steckman DA, Sauer WH, Judson MA. Pathophysiology and clinical management of cardiac sarcoidosis. Nat Rev Cardiol . 2015;12(5):278-288. doi:10.1038/nrcardio.2015.22

19. Birnie DH, Kandolin R, Nery PB, Kupari M. Cardiac manifestations of sarcoidosis: Diagnosis and management. Eur Heart J . 2017;38(35):2663-2670. doi:10.1093/eurheartj/ehw328

20. Kopriva P, Griva M, Tüdös Z. Management of cardiac sarcoidosis - A practical guide. Cor Vasa . 2018;60(2):e155-e164. doi:10.1016/j.crvasa.2017.05.012

21. Ballul T, Borie R, Crestani B, et al. Treatment of cardiac sarcoidosis: A comparative study of steroids and steroids plus immunosuppressive drugs. Int J Cardiol . 2019;276:208-211. doi:10.1016/j.ijcard.2018.11.131

22. Ashraf S, Briasoulis A, Afonso L. Infrequent cardiac manifestations of sarcoidosis. Hear Lung J Acute Crit Care . 2016;45(6):515-516. doi:10.1016/j.hrtlng.2016.08.013 
23. Kandolin R, Lehtonen J, Kupari M. Cardiac Sarcoidosis and Giant Cell Myocarditis as Causes of Atrioventricular Block in Young and Middle-Aged Adults. Circ Arrhythmia Electrophysiol . 2011;4(3):303-309. doi:10.1161/CIRCEP.110.959254

24. Birnie DH, Sauer WH, Bogun F, et al. HRS Expert Consensus Statement on the Diagnosis and Management of Arrhythmias Associated With Cardiac Sarcoidosis. doi:10.1016/j.hrthm.2014.03.043

25. Nery PB, Beanlands RS, Nair GM, et al. Atrioventricular block as the initial manifestation of cardiac sarcoidosis in middle-aged adults. J Cardiovasc Electrophysiol . 2014;25(8):875-881. doi:10.1111/jce.12401

26. Salama A, Abdullah AS, Nadkarni N, Alweis R. SARCOIDOSIS INCREASES THE RISK OF COMPLETE HEART BLOCK: A REVIEW FROM THE NATIONAL INPATIENT SAMPLE. $J$ Am Coll Cardiol . 2019;73(9):543. doi:10.1016/s0735-1097(19)31151-9

27. Nordenswan H-K, Lehtonen J, Ekström K, et al. Outcome of Cardiac Sarcoidosis Presenting With High-Grade Atrioventricular Block.Circ Arrhythm Electrophysiol . 2018;11(8):e06145. doi:10.1161/CIRCEP.117.006145

28. Ghanizada M, Rossing K, Bundgaard H, Gustafsson F. DA N I S H M E D I C A L J O U R N A L. Dan Med J . 2018;65(4):A5462.

29. SCHULLER JL, OLSON MD, ZIPSE MM, et al. Electrocardiographic Characteristics in Patients With Pulmonary Sarcoidosis Indicating Cardiac Involvement. J Cardiovasc Electrophysiol . 2011;22(11):1243-1248. doi:10.1111/j.1540-8167.2011.02099.x

30. Gheysens O, Cleemput J Van, Wuyts W. Natural evolution of cardiac sarcoidosis in an asymptomatic patient : a case report. 2019:1-6. doi:10.1093/ehjcr/ytz099

31. Segura AM, Radovancevic R, Demirozu ZT, Frazier OH, Buja LM. Granulomatous myocarditis in severe heart failure patients undergoing implantation of a left ventricular assist device. Cardiovasc Pathol . 2014;23(1):17-20. doi:10.1016/j.carpath.2013.06.005

32. Roberts WC, Roberts CC, Ko JM, Filardo G, Capehart JE, Hall SA. Morphologic features of the recipient heart in patients having cardiac transplantation and analysis of the congruence or incongruence between the clinical and morphologic diagnoses. Med (United States) . 2014;93(5):211-235. doi:10.1097/MD.0000000000000038

33. Statement on Sarcoidosis. Am J Respir Crit Care Med . 1999;160(2):736-755. doi:10.1164/ajrccm.160.2.ats4-99

34. Chapelon-Abric C, Sene D, Saadoun D, et al. Cardiac sarcoidosis: Diagnosis, therapeutic management and prognostic factors. Arch Cardiovasc Dis . 2017;110(8-9):456-465. doi:10.1016/j.acvd.2016.12.014

35. Osborne MT, Hulten EA, Singh A, et al. Reduction in $18 \mathrm{~F}$-fluorodeoxyglucose uptake on serial cardiac positron emission tomography is associated with improved left ventricular ejection fraction in patients with cardiac sarcoidosis. J Nucl Cardiol . 2014;21(1):166-174. doi:10.1007/s12350-013-9828-6

36. Hamzeh N, Steckman DA, Sauer WH, Judson MA. Pathophysiology and clinical management of cardiac sarcoidosis. Nat Rev Cardiol . 2015;12(5):278-288. doi:10.1038/nrcardio.2015.22

\section{LIST OF FIGURE LEGENDS}

Figure 1 Sinus tachycardia and left bundle branch block

Figure 2. Cardiac MRI Images showing patchy Late Gadolinium Enhancement of the basal septum and mid to apical Lateral LV wall (A), basal septum and basal Lateral wall (B) and mid inferolateral wall (C). The absence of oedema or inflammation is shown in STIR images (D) after treatment

Figure 3. Complete heart block 
Figure 4. Cardiac MRI images showing Late Gadolinium Enhancement of the mid-septum (A), basal to mid-septum and lateral LV wall (B) with corresponding oedema and inflammation in STIR images (C).

Figure 5. First degree heart block
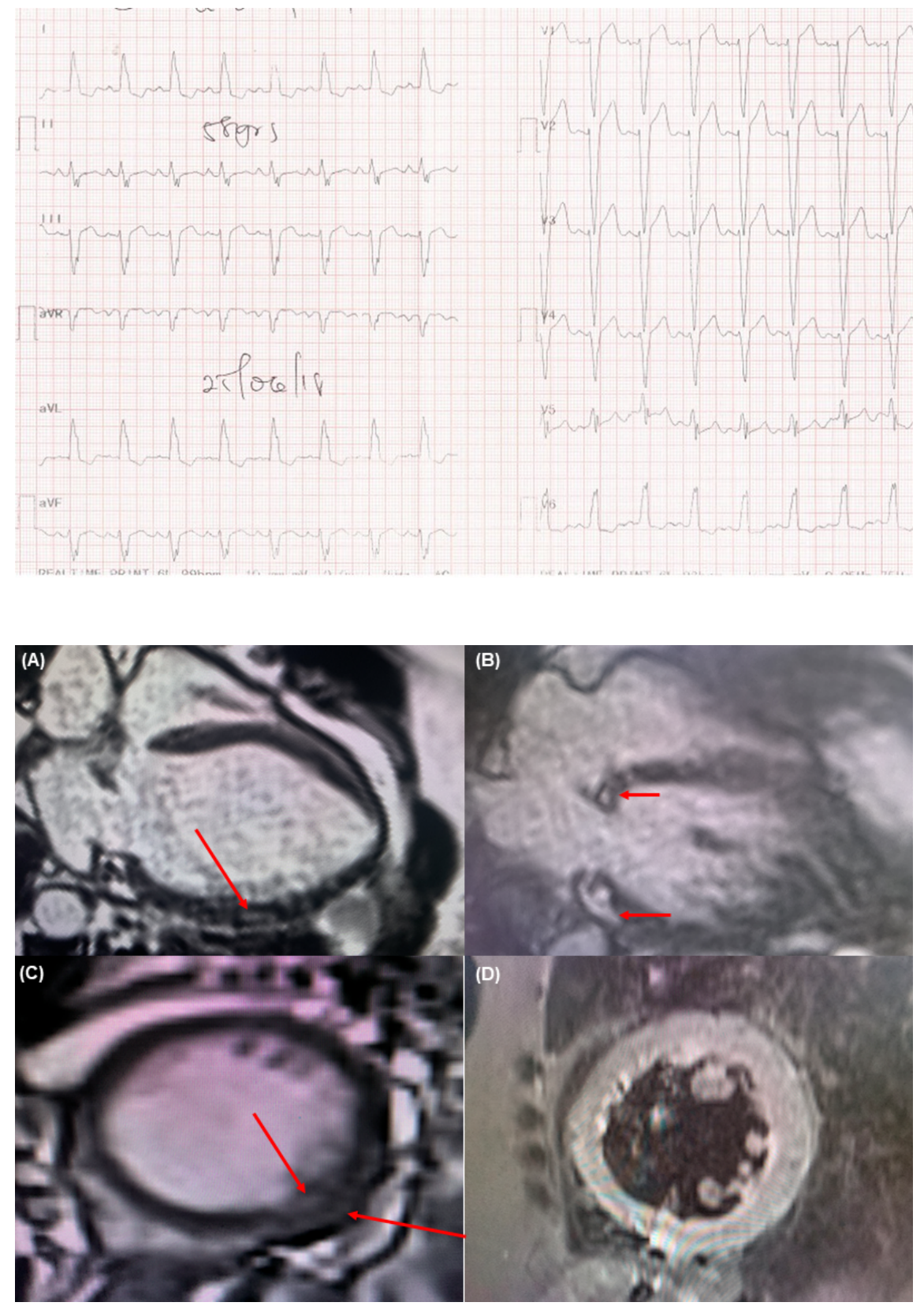

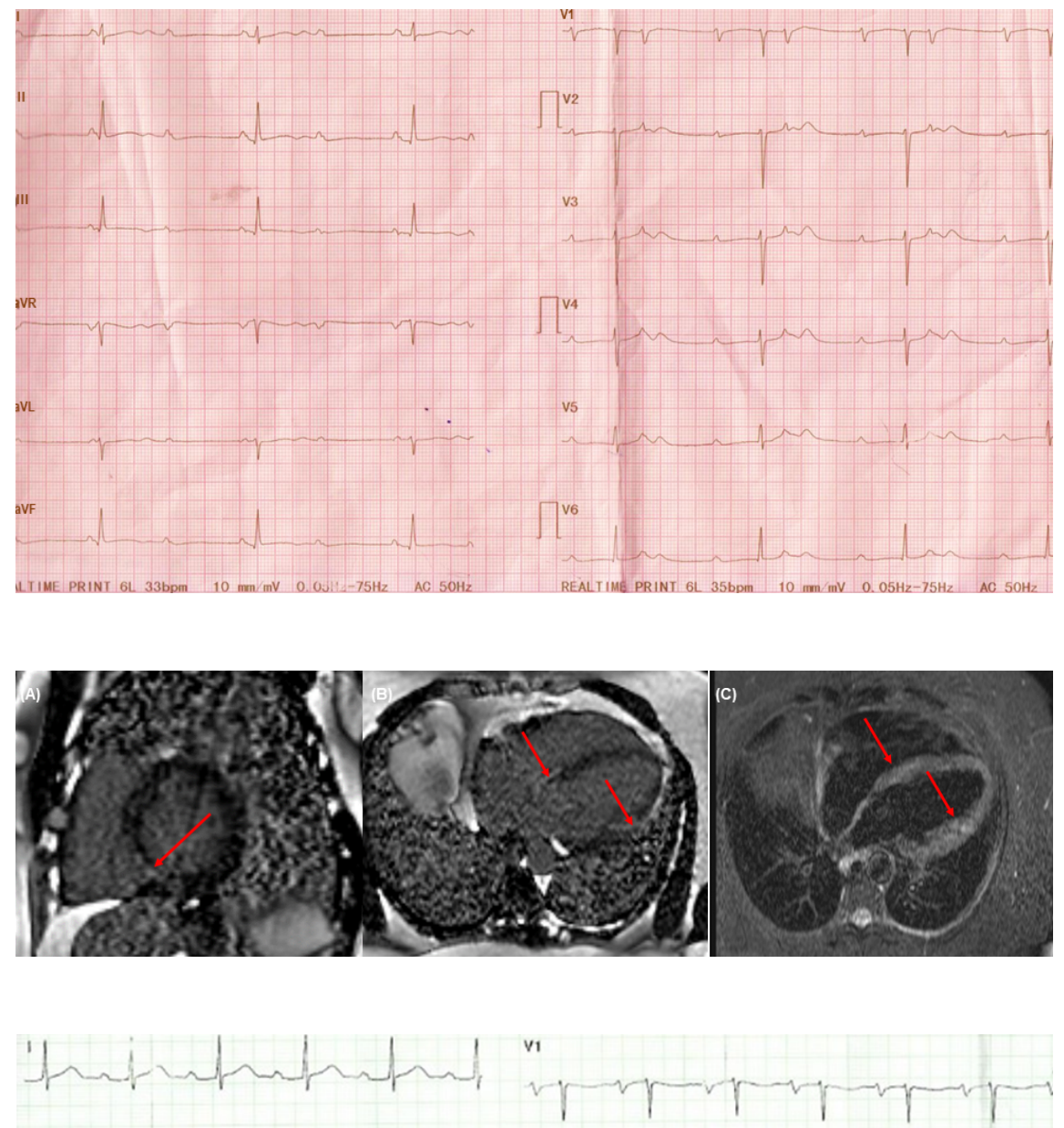

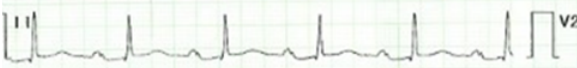

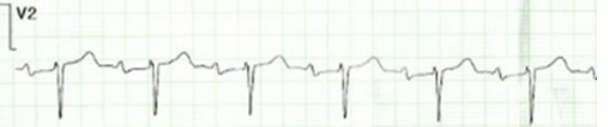

"I

v3

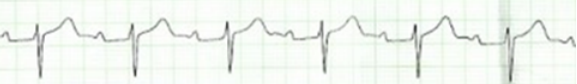
aVR $\prod^{\mathrm{v}}$

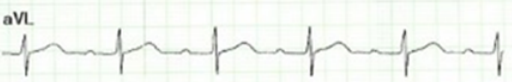

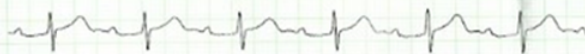
V5

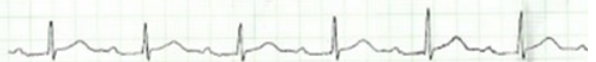

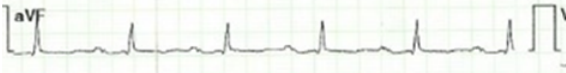
REALTIME PRINI 6L $70 \mathrm{bpm} \quad 10 \mathrm{~mm} / \mathrm{mV} \quad 0.05 \mathrm{~Hz}-75 \mathrm{H}_{2}$

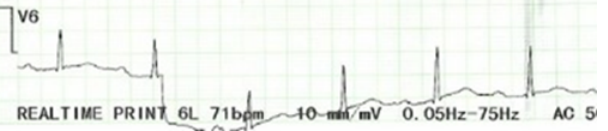

